MANY plants, particularly aquatic ones, experience periodic or even permanent waterlogging in their environment. The selective advantages conferred by morphological adaptation to such conditions is evidenced by the widespread occurrence of lacunae and large intercellular spaces within aquatic species. It has been demonstrated that oxygen penetrates along such interconnecting channels, reaching the root or rhizome extremities. For example, Coult and Vallance (J. exp. Bot. 9, 384; 1958) showed that oxygen moved the full length of a $45-\mathrm{cm}$ rhizome of Menyanthes triofoliata when it was suspended in an oxygen-free atmosphere but with the leafy shoot exposed to air.

Individual cells, nevertheless, may find themselves in conditions of low oxygen tension. Under such conditions, metabolic adaptations are essential, particularly the avoidance of ethanol accumulation as a result of anaerobic glycolysis. McManmon and Crawford (New Phytol. 70, 299; 1971) examined a range of flood-tolerant and floodintolerant species under waterlogged conditions and they found that some species, such as Pisum sativum and Vicia faba which are flood-intolerant, showed greatly increased levels of alcohol dehydrogenase (ARH, involved in acetaldehyde to ethanol conversion) under flood conditions and also high activity of 'malic' enzyme which converts malate to pyruvate (which can then be converted to acetaldehyde and hence to ethanol). Flood-tolerant species, such as Phalaris arundinacea and Glyceria maxima were found to decrease in their ADH activities as a response to flooding and showed no activity of 'malic' enzyme.

McManmon and Crawford proposed that flood-tolerant species lack malic

\section{Adaptations to waterlogged environments}

\author{
from Peter D. Moore
}

enzyme so that a diversion of glycolysis to malate production does not result in further malate decarboxylation to rejoin the path of anaerobic glycolysis and ethanol production, but ends in malate accumulation. Malate build-up seems to be harmless in these cells. In the flood-intolerant species any malate produced is decarboxylated by 'malic' enzyme, $\mathrm{ADH}$ is induced and ethanol accumulates to the detriment of the cell membranes.

Crawford and Bains (New Phytol. $79,519 ; 1977)$ have recently demonstrated that the build-up of ethanol in the roots of forest trees on waterlogging varies from one species to another. Thus, in the Sitka spruce (Picea sitchensis) which is intolerant of flooding, ethanol concentration had increased to 12 times its original level (to $5.6 \mu \mathrm{mol}$ per $\mathrm{g}$ fresh weight) after $24 \mathrm{~h}$ in anaerobic conditions, whereas in Pinus contorta, a flood-tolerant species, ethanol accumulation increased only three times its base level $(0.7 \mu \mathrm{mol}$ perg fresh weight). Once again, tolerance to flooding is associated with $a$ control of ethanol accumulation.

Crawford has now (New Phytol. 79, 511 ; 1977) examined the response of certain seeds to anoxia, for some mire plants can survive for considerable periods as seeds in anaerobic environments. For example the seeds of the rush Juncus effusus are reputed to germinate after 7 years submergence.
Sensitivity to prolonged soaking was found to vary with species. Rice seed viability was unaffected by a $6 \mathrm{~h}$ soaking, broad bean was reduced to $40 \%$ and pea was rendered completely nonviable after such periods.

In the species intolerant of soaking, such as pea and maize, ethanol was always found to be the major product of glycolysis under anaerobic conditions. Malic acid did not accumulate, but lactate was high in the early stages of glycolysis in broad bean and rice, the species tolerant of soaking. Ethanol production, however, exceeded lactate in even these species after $24 \mathrm{~h}$ soaking. The overall rate of glycolysis under anaerobic conditions was greater in the sensitive species, hence ethanol accumulated faster in these species.

Thus it would seem that the tolerance of seeds for prolonged immersion in water depends not upon any metabolic 'switch' as is the case for many roots, but upon the capacity to reduce overall metabolic rate. Crawford has further shown that for five out of six Gramineae species which he kept for 6 weeks as imbibed seeds under anaerobic conditions, there was a reduction in viability with increasing temperature. This would again suggest that increased metabolic activity leads to seed death, probably by ethanol accumulation and the associated membrane changes.

Crawford thus maintains that, although the precise metabolic techniques may vary between species and even between organs, the essential problem involved in the toleration of anaerobic conditions is the control and limitation of ethanol accumulation. $\square$ Peter D. Moore is a Senior Lecturer in the Department of Plant Sciences, King's College, London. on input through the second major afferent pathway to the hippocampus from the septum. Just what conditions lead to these plastic modulatory effects on the second input are not yet clear, however. Further research on these preparations should lead in the near future to a better understanding of plasticity in the hippocampus during learning.

The second function of the hippocampus that received considerable attention was its possible role in the detection of changed environmental circumstances, and in the behavioural adjustments that immediately follow the detection of such changes. While many participants agreed that the hippocampus is involved in the detection of change, they differed about the nature of the particular events whose change was detected. For example,
O. S. Vinogradova (USSR Academy of Sciences Biological Centre, Puschinoon-Oka) suggested that the hippocampus is involved in the detection of novelty (that is, any change in stimulus input), J. A. Gray (University of Oxford) that the hippocampus is involved in detecting changes in reinforcement schedule-in particular, from reward to non-reward, and $\mathrm{J}$. O'Keefe (University College, London), on the basis of a theory that he and L. Nadel have proposed, suggested that the hippocampus is involved in setting up spatial maps of the environment and in detecting mismatches between the environment and the map when the former is changed. Given the extent of the agreement that the hippocampus plays a part in the detection of stimulus changes, one hopes that agreement as to the nature of these changes is not too far off.

A third proposal was the hypothesis that the hippocampus is involved in certain types of spatial information processing. O'Keefe and A. H. Black (McMaster University) presented both single cell and lesion data in support of the O'Keefe and Nadel spatial theory of hippocampal function mentioned above. For example, certain hippocampal cells seem to fire only when a rat is in a particular location in a given environment, and their firing is independent of the rat's behaviour. D. Olton (Johns Hopkins University) also presented lesion data which were consistent with the view that the hippocampus is involved in spatial memory. He trained rats on an apparatus which consisted of a central circular platform from which eight arms radiated. Food pellets were placed 